\title{
Synergielösungen für Sozialräume
}

\section{Plädoyer für Fusionen kleiner Träger}

Armin Wöhrle

Es gibt Einrichtungen und Träger in der Sozialen Arbeit, die eine angemessene Größe nicht erreichen oder bereits wieder unterschritten haben. Diese instabilen Einheiten können oft keine professionelle Soziale Arbeit sichern, sondern neigen aus Überlebensgründen zu faulen Kompromissen und zur (Selbst-) Ausbeutung der Beschäftigten. Es liegt nahe, dass sich diese Träger zu einem größeren Ganzen zusammenschließen. Erfahrungen zeigen, wie Barrieren überwunden werden und Fusionen gelingen können.

Aus Initiativen entstandene kleine Träger waren schon immer und sind vom Prinzip her unterorganisiert. Die hier tätigen Menschen haben den Verein mit aufgebaut und grenzen sich gelegentlich gegen große Organisationen der Sozialwirtschaft $\mathrm{ab}$. Sie verstehen sich als Team, wollen alles, was die Arbeit betrifft, gemeinsam bereden. Der Arbeitsbereich wurde nicht selten auf dem Hintergrund von Selbsthilfe oder in enger Kooperation mit »Betroffenen « gegen Widerstände der Bürokratie und großer Träger erkämpft.

Nachdem eine minimale Grundfinanzierung durchgesetzt werden konnte, beuten sich nun die Beschäftigten oft selbst aus, um ihren Ansprüchen an Umfang und Qualität der Arbeit gerecht zu bleiben. Sie verzichten auf eine Bezahlung, die einem aufgrund der Ausbildung zustehen würde. Sie übernehmen ehrenamtlich Aufgaben zur Absicherung der Einrichtung und erwarten von jedem Mitarbeiter dasselbe. Ihre Ideen und ihre Kraft stecken in jedem Detail des Ganzen. Sie fühlen sich für alles verantwortlich (vgl. Wöhrle 1994).

Prof. Dr. Armin Wöhrle lehrt Sozialmanagement, Projektentwicklung und Beratung am Fachbereich Soziale Arbeit an der Hochschule Mittweida in Sachsen.

E-Mailwoehrle@htwm.dea
In der mit Euphorie und Mut verbundenen Aufbauphase schadet diese fehlende Organisiertheit nicht, auch Selbstausbeutung gehört dazu, allerdings lässt sich diese Phase nicht endlos verlängern. Soziale Arbeit war ein bislang expandierender Bereich (Wöhrle 2003, 59 ff.). Seit einiger Zeit gibt es Berichte, dass zumindest in der Jugendhilfe eine Trendwende einsetzt (»Erstmals kein weiterer Ausgabenanstieg im Westen - Rückgänge im Osten ", vgl. KomDat 3/2005). Wie schon in Einzelfällen, in denen der Aufbau weiterer Einrichtungen zu Kürzungen bei bestehenden führte, kann nun die Minderausstattung zur Dauerlösung werden, wenn die Beteiligten sich nicht dazu durchringen, Träger sterben zu lassen oder nach anderen Lösungen zu suchen.

Wenn fehlende Mittel anderweitig eingeworben werden müssen, kommen insbesondere kleine Träger an ihre Grenzen. Sie schaffen es gerade noch, zehn Prozent Eigenmittel (durch Einnahmen, Spenden und Sponsoring) aufzubringen, wenn Projekte durch andere Träger vorbereitet sind, können sie die zusätzliche Kraftanstrengung aufbringen, sich dort einzuklinken, jedoch fehlt ihnen die Substanz, selbst Projekte einzuwerben. Sie haben keine Grundsatzabteilung, die nach Projekttöpfen Ausschau hält und entsprechende Anträge vorbereitet. Eine Fachkraft für Fundraising können sie sich nicht leisten. Es wäre sinnvoll, ein Fundraising-Konzept trägerübergreifend anzulegen. Dafür müsste es allerdings einen angemessene Größenordnung - zumindest einen Verbund - geben.

Die gegenwärtig typische Situation der fehlenden Finanzen legt Schwächen offen, die immer schon bei kleinen Trägern vorhanden waren. Ihre Strukturschwächen aus dem Übergang einer Initiative zu einer Organisation (Wöhrle 1992, 132 ff.) werden nun jedoch offensichtlich.

Die Initiativenmitglieder haben nicht selten die Vorstände der gegründeten Vereine besetzt, um dann, wenn die Ver- eine aus öffentlichen Mitteln gefördert werden, sich selbst einzustellen. Plötzlich benötigen sie neue Vorstandsmitglieder, um formaljuristisch korrekt zu bleiben. Es werden Personen in den Vorstand gewählt, die von ihnen abhängig sein sollten, da sonst die Idee Schaden nehmen könnte. Eventuell wissen diese Vorstände gar nicht, auf welches Wagnis sie sich da beispielsweise hinsichtlich der Haftung mit dem Privatvermögen einlassen (Holt 2006). Gleichzeitig entstehen Konstruktionen, in denen Mitarbeiter als Vorstandsmitglieder gleichzeitig die Vorgesetzten ihrer Vorgesetzten sind. Oder es können Beschlüsse der Geschäftsführung und des Vorstandes durch eine Mitgliederversammlung gekippt werden, weil in der Mitgliederversammlung die Beschäftigten die Mehrheit haben.

Damit nicht genug: Organisatorische Einheiten sind manchmal so klein, dass kein Urlaub genommen werden kann, niemand krank werden darf und an Supervision und Fortbildung nicht zu denken ist. Wenn eine große Organisation einen Finanzeinbruch von zehn Prozent entweder durch zusätzliche Einwebung von Mitteln oder interner Rationalisierung fachlich unbeschadet überstehen kann, so reagiert eine zu kleine Organisation, wenn sie um jeden Preis überleben will, mit zunehmender Selbstausbeutung der Organisationsmitglieder und unweigerlichen Abstrichen an der Qualität.

\section{Mehr Angebote für die Bürger}

Dass die Fusion eine Lösung für das Dilemma mehrerer kleiner Träger sein könnte, lässt sich an einem Projekt verdeutlichen, das ich Anfang der 1990er Jahre in Stuttgart begleitete. Ausgangspunkt damals waren nicht finanzielle, sondern personelle Engpässe. Mehrere Stellen für eine Freizeitstätte konnten bei einem Träger nicht besetzt werden. Er hätte die Einrichtung dicht machen kön- 
nen und das verbleibende Personal als Springer in seinen anderen 30 Einrichtungen einsetzen können. Jedoch hatte er als mittelgroßer Träger (ca. 300 Mitarbeiter) ein Management, das unkonventionelle Wege ging.

Die zwei Personalstellen wurden den anderen beiden Trägern, die Jugendarbeit im Stadtteil betrieben unter der Bedingung zur Verfügung gestellt, dass ein gemeinsames Konzept erarbeitet würde. Dies entstand denn auch, indem mit den Ressourcen (Häusern, Räumen, Finanzen, Personal, Zeit etc.) von drei Einrichtungen flexibel umgegangen und Gesamtlösungen für den Stadtteil erdacht wurden.

Die Vision, die von den Mitarbeitern aller drei Einrichtungen entworfen wurde, durchbrach das einrichtungsbezogene Denken. Man fühlte sich für den Stadtteil zuständig. Die Konzepte der drei Einrichtungen konnten klar voneinander abge- grenzt werden (mehr betreuende, mehr selbstverwaltete Angebote, stationäre und mobile usw.) und gleichzeitig griff man neue Bedarfslagen im Stadtteil auf. Die Angebote für die Zielgruppen erhöhten sich. Die Mitarbeiter setzten sich zum Ziel, dass sich nicht nur die Qualität der Angebote, sondern auch ihre Fachlichkeit erhöhen sollte.

Ausgangspunkt hierfür war das gemeinsame Generalistentum hinsichtlich der Problemlagen von Kindern und Jugendlichen im Stadtteil und das gleichzeitige Spezialistentum jedes einzelnen Mitarbeiters und jeder einzelnen Mitarbeiterin auf unterschiedlichen Gebieten. Das konnte insbesondere dadurch ausgebildet werden, dass die Mitarbeiter einrichtungsübergreifend tätig werden konnten. Sie waren stationär und mobil tätig, konnten also Fälle auch außerhalb der Einrichtungen betreuen.

\section{Fragen: Sind wir reif für eine Fusion?}

1 Sind in Ihrer Organisation die Geschäftsführungsaufgaben von den Vorstands1 - aufgaben und von den Aufgaben der Mitarbeiter personell eindeutig getrennt?

2 Ist Ihre Organisation personell so ausgestattet, dass die Vertretung im Krank2 •heits- und Urlaubsfall gesichert ist?

2 Ist der Austausch unter Professionellen (Fachpersonal der Sozialen Arbeit) in 3 - Ihrer Organisation gewährleistet?

$\triangle$ Stehen für Supervision und Fortbildung ausreichend Geldmittel zur Verfügung und ๑kann die Teilnahme daran in der Arbeitszeit abgesichert werden?

5.

- Wird in Ihrer Organisation nach Leistung entlohnt?

6. Konnten eventuelle Kürzungen von Zuschussmitteln in Ihrer Organisation durch die Einwerbung von anderweitigen Mitteln aufgefangen werden?

7 Sind ehrenamtliche Aufgaben von professionellen Aufgaben eindeutig unter-schieden?

Sollten Sie fast alle Fragen mit einem Nein beantwortet haben, müssten Sie überlegen, ob Ihr Träger eine überlebensfähige Größe aufweist oder ob es nicht besser ist, über eine Fusion nachzudenken. Armin Wöhrle

\section{Den Wandel managen}

Seit der Begriff Sozialmanagement eingeführt wurde, wird auch vom Umbau der Organisationen Sozialer Arbeit gesprochen. Der Wandel der Sozialwirtschaft ist eng mit der Qualifikation des Managements verbunden, da die Qualität des Managements entscheidend ist, wenn die Überlebensfähigkeit der Organisation in turbulenten Zeiten gesichert werden soll. Der Band aus dem Studienkurs »Management in der Sozialwirtschaft « behandelt die zentralen Fragestellungen des Wandels und wie er aus Sicht des Managements zu bewerkstelligen ist. In einem Kapitel wird ausführlich auf Organisationsberatung und das Verhältnis zwischen Management und Beratung eingegangen. Der Autor gibt Anregungen, auf was zu achten ist, wenn Beratung ausgewählt und ein Kontrakt geschlossen wird. Konzipiert als Lehrbuch, enthält der Band Beispiele, Kontrollfragen und Übungsaufgaben, die auch dem Studierenden und dem Praktiker nützlich sein können.

Armin Wöhrle: Den Wandel managen. Organisationen analysieren und entwickeln. Nomos Verlagsgesellschaft, Baden-Baden 2005. 189 Seiten. 19,80 Euro. ISBN 3-8329-1059-X.

Im Ergebnis brachte das Projekt mehr für den Stadtteil, mehr für die Kinder und Jugendlichen und mehr für die Mitarbeiter. Die signifikant erhöhte Mitarbeiterzufriedenheit äußerte sich auch darin, dass es zukünftig keine offenen Stellen in diesem Stadtteil mehr gab. Das ursprünglich aus einer Mangelsituation entsprungene Projekt wurde richtungsweisend für die Kommune und darüber hinaus. Die beteiligten Träger konnten einen Imagegewinn verbuchen.

Bei Projektende waren die Vorteile eindeutig belegt. Die 36 Ergebnisziele wurden anhand von 164 Teilaspekten ausgewertet (Wöhrle 1997). Dennoch sperrten sich zwei der drei Träger gegenüber einer großen organisationalen Lösung, die darin bestanden hätte, alle drei Einrichtungen unter das Dach eines neu zu gründenden Trägers (einer gGmbH, an der alle bisherigen Träger einen Anteil gehalten hätten) zu überführen. Interessanterweise sperrte sich nicht der große Träger - es waren die beiden kleinen.

Im Gegensatz zu den kleinen Trägern verfügen große Organisationen der Sozialwirtschaft mit mehreren hundert oder sogar tausend Beschäftigten über ein professionelles Management, das die Vorund Nachteile von Kooperationen und Fusionen klären oder zur Klärung in Auftrag geben kann. Die großen Träger gehen somit Fusionen aus rationalen Gesichtspunkten an.

Für die Organisationsmitglieder kleiner Träger ist die Vorstellung einer Fusion bedrohlich. Da sie nicht als Übernahme denkbar ist, ist sie gleichbedeutend mit einem Identitätsverlust hinsichtlich der eigenen Organisation. Alles, woran das Herzblut hängt, was man sich in einer bestimmten, unverwechselbaren Art und Weise eingerichtet hat, könnte aufgegeben werden müssen. Es gibt keine Sicherheit, dass das Neue noch von einem selbst in den wesentlichen Punkten beeinflusst werden kann.

Die Vorstellung schreckt, dass die Ökonomie die sozialen Werte determinieren könnte. Manchmal sind es auch Vorurteile gegenüber anderen Trägern, die eine Kooperation behindern. Die Befürchtungen sind so stark, dass die Selbstausbeutung und die unbefriedigenden Bedingungen für eine fachliche Arbeit lieber in Kauf genommen werden, als sich auf ein unsicheres Terrain zu begeben, auf dem nach rationalen Gesichtspunkten verhandelt wird. Widerstände haben selten fachlich- 
rationale Gründe. Es ist ohnehin besser zu erklären, weshalb sich Organisationen nicht wandeln als zu erklären, weshalb sie es tun (vgl. Wöhrle 2002, 62 ff.).

Was würde kleinen Organisationen helfen, Schritte zu einer Fusion mit anderen zu wagen? Ich spreche hier nicht davon, sich einem großen Träger zur Einverleibung anzubieten, sondern von der Verhandlung mit mehreren kleinen Trägern über eine selbst verantwortete Fusion. Helfen würde ihnen, wenn sie sich eine externe Organisationsberatung leisten könnten. Schon ihre Finanzierung könnte der Test sein: Jeder einzelne Träger könnte sie sich nicht leisten, aber alle zusammen? Manchmal gibt es Organisationsberatung im Rahmen größerer Projekte (vgl. Wöhrle 2005a) auch finanziert und manchmal kann man sich speziell von Stiftungen, wenn Innovation angestrebt wird, Beratung finanzieren lassen.

Gehen wir davon aus, dass eine externe Beratung zustande käme und diese hierfür passend ausgewählt worden wäre, so können sich folgende Möglichkeiten eröffnen:

- Aus den Ressourcen mehrerer Träger und wenn die Organisationsmitglieder entsprechend mitspielen -, kann sich eine neue Trägerkonstruktion ergeben, die den Finanz- und Auftraggebern gewichtiger gegenübertritt.
- Eine Organisationsentwicklung, die die jeweils innovativen Kräfte der beteiligten Träger bündelt, kann neue fachliche Konzepte befördern, die Synergielösungen für Sozialräume erbringen.

- Engepässe an Ressourcen (Finanzen, Personal, Räume, Zeit usw.) können produktiv überwunden werden.

- Für die Mitarbeiter und die Verantwortlichen können sich befriedigendere Aufgaben- und Tätigkeitsbereiche ergeben. Wichtig für den Prozess der Fusionierung ist allerdings, dass eine professionelle Organisationsentwicklung (Wöhrle 2005 b) initiiert und begleitet wird, in deren Gefolge

- alle Organisationsmitglieder einbezogen, umfassend informiert und an den Entscheidungen angemessen beteiligt werden,

- Befürchtungen hinsichtlich Werteverluste, verschlechterten Arbeitsbedingungen oder Arbeitsplatzverlust Ernst genommen werden,

- neue gemeinsame Werte, Regeln und Verfahrensweisen erfunden und verbindlich werden,

- Machtspiele und Vorurteile nicht angeheizt werden,

- ein anerkanntes Management institutionalisiert wird,

- neuen fachlichen Lösungen Raum gegeben wird,

\section{Das etwas andere Management}

Management wurde, so scheint es, für Organisationen der Sozialen Arbeit in den 1980er Jahren »entdeckt«. Seitdem ist über Sozialmanagement viel geschrieben worden. Eine seriöse Annäherung nimmt Bezugspunkte in den Blick und lotet dann aus, was hinsichtlich des neuen Phänomens sichtbar ist. So wird auch in diesem Band verfahren. Es muss zur Kenntnis genommen werden, dass es Organisationstheorien und eine Managementlehre bereits gab, bevor in der Sozialen Arbeit über Sozialmanagement diskutiert wurde. Der zweite Bezugspunkt ist das sozialpolitische und wirtschaftliche System, in das soziale Dienstleistungen eingebunden sind, über das aus volkswirtschaftlichem und sozialpolitischem Blickwinkel, aus Sicht eines dritten Sektors, von Non-Government- und Non-Profit-Organisationen, Organisationen der Sozialen Arbeit und der Sozialwirtschaft diskutiert wird. Der dritte Bezugspunkt ist die Soziale Arbeit in ihrer Fachlichkeit. Sie wird in diesem Buch aus dem Studienkurs »Management in der Sozialwirtschaft « als zentrale Voraussetzung unterstellt, da sie für die Ausrichtung des Managens und für die Qualität der Dienstleistungserbringung unverzichtbar ist. Ausgerichtet an den Bestimmungen der Managementlehre gibt es weitreichende Übereinstimmungen, aber in den praktischen Bezügen fallen Besonderheiten hinsichtlich des Managements in der Sozialwirtschaft auf. Es handelt sich offensichtlich um ein etwas anderes Management. Hieraus ergeben sich nun auch Anforderungen an die Qualifikation des Managements, an die Ausbildung und an eine entsprechende Forschung. Letztlich stellt sich die Frage nach der theoretischen Einordnung des Managements in der Sozialwirtschaft in das Wissenschaftsgefüge.

Armin Wöhrle: Grundlagen des Managements in der Sozialwirtschaft, Nomos Verlagsgesellschaft, Baden- Baden 2003. 191 Seiten. 19,80 Euro. ISBN 3-8329-0341-0.

- Konfliktmanagement

professionell praktiziert wird,

- für notwendige zusätzliche Arbeitsbelastung Ausgleich geschaffen und

- Engagement belohnt wird.

Fusionen kleiner Träger können für einen Stadtteil einen signifikanten Mehrwert bedeuten. Und sie können die $\mathrm{Zu}$ friedenheit und die Stellensicherheit für die Mitarbeiter signifikant erhöhen. Und die beteiligten Organisationen können durch höhere Qualität und mehr Angebote ein besseres Ansehen erwarten.

Fazit: Das oft aus einer Krise heraus begonnene Projekt einer Verbindung zweier oder mehrerer Organisationen auf gleicher Augenhöhe verspricht bei kompetenter Beratung zu einem Gewinn für alle zu werden.

\section{Literatur}

KomDat - Kommentierte Daten der Kinder- und Jugendhilfe, Informationsdienst der Dortmunder Arbeitsstelle Kinder- und Jugendhilfestatistik (Dezember 2005) 3/2005.

von Holt, Th.: Persönliche Haftungsrisiken von Vorstandsmitgliedern und Vereinsgeschäftsführern. http://www.sozialbank.de/finale/inhalt/servicel/fachbeitraege32223.shtml [April 2006] Wöhrle, A.: Jugendhilfe und Management. Fortbildung und Beratung im Kontext von Personal- und Organisationsentwicklung, München 1992.

Wöhrle, A: »Wir sind alle gleich ...!? « Steuerungstabus und Organisationsentwicklungserfordernisse in alternativen Projekten und bei kleinen Trägern, S. 30 ff., in: Sozialmagazin 6/1994.

Wöhrle, A.: »echt! « Elegante Lösungen für: Bedarfsorientierung, Vernetzung und Professionalisierung. Ein Bericht über das Projekt in Stuttgart-Fasanenhof, S. 19, in: Sozialmagazin 7-8/1997. Wöhrle, A.: Change Management. Organisationen zwischen Hamsterlaufrad und Kulturwandel, Augsburg 2002.

Wöhrle, A.: Grundlagen des Managements in der Sozialwirtschaft, BadenBaden 2003.

Wöhrle, A: Organisationsberatung und Coaching als Beiträge zur Stärkung der Sozialwirtschaft. Internet http://www. equal-sachsen-sozialwirtschaft.de/download/ZZZModul_Woehrle.pdf (2005a) [Januar 2005]

Wöhrle, A.: Den Wandel managen. Organisationen analysieren und entwikkeln, Baden-Baden 2005b. 